\title{
PREVALENCIA DE LAS ECTASIAS CORNEALES EN LA CLÍNICA OFTALMOLÓGICA SOLEX LTDA. EN EL PRIMER SEMESTRE DE 2013 SUCRE, BOLIVIA
}

\author{
${ }^{1}$ Álvaro Ernesto Martínez Izquierdo, ${ }^{2}$ Freddy Guerra Paniagua, ${ }^{3}$ Glynka María Guerra Torrico, ${ }^{3}$ Kerly Dayana Ibáñez Felizzola, \\ ${ }^{3}$ Judith Alicia Cárdenas Remolina
}

\begin{abstract}
${ }^{1}$ Optómetra Esp. en Segmento Anterior y Lentes de Contacto. Docente U. Santo Tomás Seccional Bucaramanga ${ }^{2} \mathrm{Md}$. oftalmólogo Esp. en Segmento Anterior y Cirugía Refractiva. Clínica Oftalmológica Solex, Sucre, Bolivia ${ }^{3}$ Estudiantes $X$ semestre de Optometría. U. Santo Tomás Seccional Bucaramanga
\end{abstract}

Autor responsable de correspondencia: Álvaro Ernesto Martínez Izquierdo

Correo electrónico: alvaro-emi@hotmail.com

\begin{abstract}
RESUMEN
Objetivos: determinar la prevalencia y caracterización de ectasias diagnosticadas por topografía corneal en la Clínica Oftalmológica Solex Ltda. en el primer semestre de 2013 Sucre, Bolivia.

Materiales y métodos: estudio observacional descriptivo de corte transversal. La población fueron los pacientes con topografia corneal en el primer semestre del 2013 en la Clínica Oftalmológica Solex Ltda.

Resultados: de 170 pacientes que entraron al estudio el 57,6\% son del género femenino y 42,6\% masculino. La edad mínima fue de 7 años y la máxima de 64 años con un promedio de 28,2 años; presentándose mayor frecuencia los pacientes de 30 años [13 pacientes, $7,6 \%$. De acuerdo con el patrón topográfico se evidencia que el 57,2\% corresponde a la forma irregular, valor de gran significancia. La de menor presentación corresponde al corbatín asimétrico [14 presentaciones].

Conclusiones: en el presente estudio se encontró una diferencia entre el género femenino [57,6\%] y masculino [42,4\%] del 15,2\%. En cuanto a la edad su distribución muestra un rango comprendido entre los 7 y 64 años. De las 339 topografias que se realizaron 279 tuvieron una excelente toma y 60 presentó una calidad reducida debido a falta de anillos de placido, sin embargo, el equipo los clasifica con un $97 \%$ de área analizada [normal]. La prevalencia de ectasias corneales en la Clínica Oftalmológica Solex Ltda. de Sucre, fue del $57,2 \%$. En ojo derecho $47,9 \%$ y del $52,1 \%$ en ojo izquierdo sobre un total de 339 exámenes, es importante destacar que es una prevalencia alta. [Martínez AE, Guerra F, Guerra GM, Ibáñez KD, Cárdenas JA. Prevalencia de las ectasias corneales en la Clínica Oftalmológica Solex Ltda. En el primer semestre de 2013, Sucre, Bolivia. Ustasalud. 2014;13(2): 151 - 156]
\end{abstract}

Palabras clave: Ectasia, Topografía corneal, Observaciones clínicas.

\section{PREVALENCE OF CORNEAL ECTASIAS IN SOLEX LTDA. OPHTHALMOLOGICAL CLINIC DURING FIRST SEMESTER OF 2013 SUCRE - BOLIVIA}

\footnotetext{
ABSTRACT

Objective: to determine the prevalence and characterization of ectasias diagnosed by corneal topography in the Eye Clinic Solex Ltda. [Sucre - Bolivia] in the first half of 2013.

Methods: a descriptive cross-sectional observational study. The population were patients with corneal topography in the first half of 2013 at the Eye Clinic Solex Ltda.

Results: of 170 patients who entered the study $57.6 \%$ were female and $42.6 \%$ male. The minimum age was 7 years and maximum of 64 years with an average of 28.2 years; appearing more frequently patients 30 years [13 patients - 7.6\%]. According to the topographic pattern it shows that $57.2 \%$ corresponds to the irregular shape, value of great significance. The lower display corresponds to the asymmetric bowtie [14 presentations].

Conclusions: in this study a difference between female gender [57.6\%] and men [42.4\%] of $15.2 \%$ was found. In terms of age distribution shows a range between 7 and 64 years. Of the 339279 topographies that were made they had a great shot and 60 present a reduced quality due to lack of placido rings, however the team classifies with $97 \%$ of analyzed area [normal]. The prevalence of corneal ectasia in the Eye Clinic Solex Ltda. of Sucre, was $57.2 \%$. Law $47.9 \%$ and $52.1 \%$ in the left eye on total of 339 tests, eye is important to note that it is a high prevalence
}

Key words: Eecstasies, corneal topography, clinical observations

Recibido para publicación: 16 de agosto de 2014. Aprobado para publicación: 3 de Octubre de 2014 


\section{INTRODUCCIÓN}

Las ectasias corneales se caracterizan por un aumento progresivo de la curvatura de la córnea que ocurre generalmente de manera asimétrica; causando una serie de síntomas como deslumbramientos, fotofobia, irritación, visión borrosa, y visión doble, dificultando la corrección con gafas y haciéndose indispensable el uso de lentes de contacto y en casos más avanzados se hace necesario los procedimientos quirúrgicos [anillos intraestromales, entrecruzamiento de colágeno, queratopatía penetrante] llegando a comprometer significativamente la salud visual de la población afectada. Los factores etiológicos fundamentales de estas distrofias son genéticos y ambientales, este último causante de la alergia ocular, el cual va acompañado del hábito de frotarse los ojos que resulta siendo significativo para la aparición de dicha patología, especialmente para el queratocono ${ }^{1}$

En el ambito mundial, la prevalencia de las ectasias corneales es altamente variable, por tanto, su compromiso visual varía según la región, adicionalmente en cuanto a factores ambientales, diversos estudios indican que los pacientes con ectasia corneal, en general, se frotan más los ojos, que los pacientes control, en otro estudio se encontró que la prevalencia del queratocono en Jerusalén es mucho mayor que la observada en otras partes del mundo, a excepción de la India, lo cual puede también estar relacionado con una combinación de factores genéticos y ambientales ${ }^{2}$.

Sucre ubicada en el departamento de Chuquisaca, Bolivia, esta población se encuentra a 2800 de altura msnm, lo cual provoca mayor exposición a los rayos $\mathrm{UV}^{3}$, acompañada de un clima templado, la unión de estas condiciones geográficas produce una asociación importante entre las ectasias con la alergia ocular y el frote ocular ${ }^{4,5}$; estas manifestaciones despertaron el interés de tres oftalmólogos por conocer más acerca de la prevalencia y caracterización de dicho grupo de patologías, ya que la información es limitada en este medio.

En atención a la problemática, surgió la necesidad de establecer la prevalencia de ectasias corneales en la Clínica Oftalmológica Solex Ltda., ubicada en Sucre, Bolivia realizando el diagnóstico topográfico. Este interés nació de la práctica de sus profesionales (tres oftalmólogos), quienes han encontrado una alta frecuencia de estas patologías, sobre todo en pacientes interesados en cirugía refractiva; sin embargo, no hay estudios, ni otros datos demográficos o estadísticos que cuantifiquen la magnitud del problema, dando origen a las siguientes preguntas ¿cuál es la frecuencia de ectasias corneales diagnosticadas por topografía en la Clínica Solex de la ciudad de Sucre en el primer semestre 2013? y ¿cuáles son las características topográficas de las mismas?.

\section{MATERIALES Y MÉTODOS}

Se planteó la realización de un estudio observacional descriptivo de corte transversal, ya que no se efectuó ninguna intervención a los pacientes. Se hizo una medición de prevalencia descriptiva de las ectasias corneales, el tipo de ectasia y el grado de afectación de la córnea, teniendo en cuenta los índices del topógrafo Galilei.

Se realizó un análisis completo de las topografias corneales, teniendo en cuenta las siguientes variables: patrón topográfico, SIMK1-2-PROMEDIO, astigmatismo, paquimetría central y delgada, KPI (índice de predicción del queratocono), KPROB (índice de probabilidad del queratocono) e índices del queratocono (I-S, SAI, SRI, IAI, AA, DSI, OSI, CSI, ACP, SDP), con el fin de definir los exámenes con y sin ectasias. El análisis estadístico se efectuó con el programa EpiInfo.

\section{Implicaciones bioéticas}

Según la Resolución 008430 del 4 de octubre de 1993. Esta investigación se clasifica sin riesgo: ya que es un estudio observacional descriptivo, y no se hizo ningún tipo de intervenciones con los pacientes, se trabajó con una base de datos de topografias de los pacientes de la Clínica Solex Ltda, Sucre, Bolivia.

En Bolivia no se tiene regulación ética para este tipo de estudio, lo que se encontró fue el Código de Ética y Deontología Médica del Ministerio de Salud de la República de Bolivia, en el cual se establecen las pautas para la atención e investigación en salud ${ }^{6}$.

La investigación traerá consigo beneficios para la institución, ya que proporcionará una mejor toma de decisiones al protocolo quirúrgico.

La Clínica Oftalmológica Solex Ltda tiene derecho a decidir de forma autónoma y libre su participación para esta investigación y la aceptación voluntaria de acceder a la base de datos para su respectivo análisis y podía retirarse de la investigación cuando lo desee.

El manejo de la confidencialidad de los datos se llevó a cabo asignando un código sin revelar el nombre del paciente.

\section{Selección y descripción de participantes}

La población equivale a los pacientes a los cuales se les realizó topografias corneales para el primer semestre de 2013 en la Clínica Oftalmológica Solex Ltda. Se tomó el $100 \%$ de las topografías que cumplían con los criterios de selección, el muestreo no aplica, puesto que la muestra es el $100 \%$ de la población.

Se tuvo en cuenta la primera topografia registrada el primer semestre de 2013 en el topógrafo Galilei. Los criterios de selección de los participantes se tomaron en cuenta de acuerdo con los criterios de inclusión y 
exclusión. Dentro de los criterios de inclusión se encuentran:

- Pacientes que se encuentren registrado en la base de datos del topógrafo Galilei de la Clínica Oftalmológica Solex Ltda, con exámenes realizados en el primer semestre de 2013.

- Se excluyeron las topografías de pacientes que tengan patrones que representen cirugías refractivas.

\section{RESULTADOS}

\section{Análisis univariado}

De 170 pacientes que entraron al estudio se encontró un porcentaje del $57,6 \%$ representado por el género femenino y $42,4 \%$ por el género masculino.

La edad mínima fue de 7 años y la máxima de 64 años con un promedio de 28,2 años; presentándose una mayor frecuencia en los pacientes de 30 años que corresponde al 7,6\% (13 pacientes).

De acuerdo con el patrón topográfico se evidencia que el $57,2 \%$ corresponde a la forma irregular, valor de gran significancia. La de menor presentación corresponde al corbatín asimétrico con una frecuencia de 14 presentaciones.

Del total de las topografías del estudio que se clasificaron como irregulares todas corresponden a ectasias corneales (Tabla 1).

En cuanto al tipo de ectasia se evidencia que la de mayor presentación corresponde a la ectasia incipiente con 121 casos $(62,4 \%)$ y la de menor presentación es degeneración marginal pelúcida con un 2,6\% (Tabla 2).

Se observa en el grupo de no ectasia que el Simk K1 predominan los valores planos con una frecuencia del $55,9 \%$.

En los pacientes con ectasia la mayor frecuencia la tienen las córneas con valores normales $(46,9 \%)$.

En el grupo de no ectasia se encontró que la Simk K2 de mayor frecuencia corresponde a los valores curvos, con un porcentaje del $76,6 \%$ y solo el $0,7 \%$ corresponde a córneas planas. Del grupo de sí ectasia no se encuentran valores planos para Simk K2.

El valor de la queratometría simulada (SimK promedio), en pacientes de córneas sanas presentan un promedio de 43,87 D. En pacientes con ectasia corneal el promedio es de $46,10 \mathrm{D}$.

El espesor central presenta como mayor frecuencia para el caso de no ectasias los normales, 71 casos $(49,0 \%)$ y para los de si ectasia los reducidos $42,3 \%$ (82 casos).

Así mismo, tres exámenes no presentaban datos de paquimetría delgada, los cuales se encuentran clasificados en el grupo de sí ectasia.
Se puede observar en el grupo de pacientes sin ectasia corneal paquimetrías delgadas con valores normales del 51,0\%. En cuanto al grupo de pacientes que sí presentan ectasia corneal se observa mayor frecuencia de valores reducidos $(57,6 \%)$.

El KPI en el grupo de no ectasia presenta su mayor frecuencia en los valores de normal con un 93,1\% y una menor frecuencia para queratocono $(2,8 \%)$. Para el grupo de sí ectasia la menor frecuencia corresponde al valor de sospechoso $(7,7 \%)$ y una mayor frecuencia para el grupo de normal $(63,4 \%)$ (Tabla 3).

El Kprob en el grupo de no ectasia se presentan 7 casos con queratocono, el cual corresponde al 4,8\% y para el grupo de sí ectasia la mayor frecuencia corresponde al valor normal $(64,9 \%)$ (Tabla 4).

\section{Análisis bivariado}

Todas las variables presentaron valores de coeficiente de asimetría superiores a 0 y para el caso de la curtosis sus valores fueron superiores a 0,263 , lo cual hace que todas sean consideradas no normales.

Del total de la población, la edad mínima 7 años y la máxima 64 años, se encuentran presentes en el grupo de no ectasia.

La mediana para ambos casos es muy similar.

Para la población de ectasia, el valor mínimo de SimK k1 fue 39,55 D y el máximo de 56,64 D. En el grupo de no ectasia se observa un valor mínimo de $38,07 \mathrm{D}$ y el máximo de 45,99 D, mostrando una variación de $10 \mathrm{D}$ en los valores máximos.

El Simk K2 muestra diferencia de 17 D entre los valores máximos encontrados entre los grupos de no y sí ectasia. Con una mediana muy similar.

Para el grupo de ectasia la mediana es $45,12 \mathrm{D}$, y para el grupo de sin ectasia es de 43,84 D y en total es de 44,48 en el SimK promedio.

Para el grupo de ectasia se observa una paquimetría central mínima de $389 \mu \mathrm{m}$ y una máxima de $640 \mu \mathrm{m}$, con una mediana de $526,5 \mu \mathrm{m}$ y para el grupo de sin ectasia un valor mínimo de $461 \mu \mathrm{m}$ y un valor máximo de $667 \mu \mathrm{m}$.

Por otra parte, la paquimetría más delgada presenta una mediana de $514 \mu \mathrm{m}, 539 \mu \mathrm{m}$ y $528 \mu \mathrm{m}$, en los grupos de ectasia, no ectasia y total respectivamente.

KPI tiene una mediana de 0,25 para el grupo de ectasia y un valor de 0 para el no ectasia y para el total (Tabla 5).

El Kprob presenta una mediana de 2,2 en el grupo de ectasia, 0,3 en el de con ectasia y en total del grupo 1,0 (Tabla 6). 
ARTÍCULO DE INVESTIGACIÓN CIENTÍFICA Y TECNOLÓGICA

Tabla 1. Presencia de ectasia corneal

\begin{tabular}{lcccc}
\hline & ECTASIA & Frecuencia & Porcentaje & \multicolumn{2}{c}{ 95\% Conflimits } \\
\hline No & 145 & $42,8 \%$ & $37,5 \%$ & $48,2 \%$ \\
Si & 194 & $57,2 \%$ & $51,8 \%$ & $62,6 \%$ \\
Total & 339 & $100,0 \%$ & & \\
\hline
\end{tabular}

Tabla 2.Tipo de ectasia corneal según patrón topográfico

\begin{tabular}{lcccc}
\hline \multicolumn{1}{c}{ TIPO DE ECTASIA } & Frecuencia & Porcentaje & \multicolumn{2}{c}{ 95\% Conflimits } \\
\hline Degenera. marginal pelúcida & 5 & $2,6 \%$ & $0,8 \%$ & $5,9 \%$ \\
Ectasia incipiente & 121 & $62,4 \%$ & $55,1 \%$ & $69,2 \%$ \\
Queratocono & 68 & $35,1 \%$ & $28,4 \%$ & $42,2 \%$ \\
Total & 194 & $100,0 \%$ & & \\
\hline
\end{tabular}

Tabla 3. KPI en categorías

\begin{tabular}{lcccc}
\hline \multirow{2}{*}{ KPI } & \multicolumn{2}{c}{ No ectasia } & \multicolumn{2}{c}{ Si ectasia } \\
\cline { 2 - 5 } & Frecuencia & Porcentaje & Frecuencia & Porcentaje \\
\hline Normal & 135 & $93,1 \%$ & 123 & $63,4 \%$ \\
Queratocono & 4 & $2,8 \%$ & 56 & $28,9 \%$ \\
Sospechoso & 6 & $4,1 \%$ & 15 & $7,7 \%$ \\
Total & 145 & $100,0 \%$ & 194 & $100,0 \%$ \\
\hline
\end{tabular}

Tabla 4. Kprob en categorías

\begin{tabular}{ccccc}
\hline \multirow{2}{*}{ Kprob } & \multicolumn{2}{c}{ No ectasia } & \multicolumn{2}{c}{ Si ectasia } \\
\cline { 2 - 5 } & Frecuencia & Porcentaje & Frecuencia & Porcentaje \\
\hline Normal & 137 & $94,5 \%$ & 126 & $64,9 \%$ \\
Queratocono & 7 & $4,8 \%$ & 61 & $31,4 \%$ \\
Sospechoso & 1 & $0,7 \%$ & 7 & $3,6 \%$ \\
Total & 145 & $100,0 \%$ & 194 & $100,0 \%$ \\
\hline
\end{tabular}

Tabla 5. KPI

\begin{tabular}{lccc}
\hline & \multicolumn{3}{c}{ KPI } \\
\hline Grupo & Ectasia & Sin ectasia & Total \\
\hline Mediana & 0,25 & 0 & 0 \\
Mínimo & 0 & 0 & 0 \\
Máximo & 100 & 100 & 100 \\
\hline
\end{tabular}

Tabla 6. Kprob

\begin{tabular}{lccc}
\hline & \multicolumn{3}{c}{ Kprob } \\
\hline Grupo & Ectasia & Sin ectasia & Total \\
\hline Mediana & 2,2 & 0,3 & 1 \\
Mínimo & 0 & 0 & 0 \\
Máximo & 100 & 100 & 100 \\
\hline
\end{tabular}

154 


\section{DISCUSIÓN}

En el estudio de Ramón García Pérez "Estudio retrospectivo sobre 290 casos de queratocono ${ }^{7}$ se encontró que la presencia de ectasia corneal es mayor en hombres que mujeres, considerándolo como un importante problema de salud pública en España, en el que el sexo masculino es un factor de riesgo. En el presente estudio esto no se da, ya que predominó el género femenino sobre el masculino, pero es importante resaltar que en el estudio hay más personas del género femenino.

En el estudio "Características topográficas del queratocono en nuestro medio" de Adel y colaboradores en $\mathrm{Cuba}^{8}$, encontraron que los pacientes del grupo de queratocono eran más jóvenes que los pacientes normales, tampoco se presentó coincidencia con esta información, ya que no hay diferencia en la edad entre el grupo normal y con queratocono.

En el estudio "Corneal elevationindices in normal and keratoconiceyes" de Fam y Lim, en los hospitales de Singapur y Malasia ${ }^{9}$, plantean que los índices tanto de elevación anterior como posterior presentan valores elevados o por fuera de la normalidad en el caso de las ectasias. En el caso del presente estudio los datos obtenidos coinciden con esto, ya para la elevación anterior se encontraron que el 97,9\% presentaron valores alterados y en el caso de la elevación posterior fue del 99,5\%. De igual forma, en los índices de elevación anterior y posterior concluyen que hubo diferencias significativas entre los grupos normales y el grupo de queratocono. En este punto lo encontrado en el presente estudio arroja valores que no muestran diferencia significativa entre estos índices.

En el estudio de "Comparison of and correlation between anterior and posterior corneal elevation maps in normal eyes and keratoconus-suspecteyes" de Schlegel y colaboradores en Francia ${ }^{10}$ tomaron 14 índices generados por el Orbscan, para comparar y correlacionar entre ojos normales y ojos sospechosos de queratocono, concluyendo que si hay 8 o más índices alterados corresponde a ojos con sospecha de queratocono. El presente estudio se trabajó sobre 23 indices del Galilei, encontrando que existe acuerdo con lo planteado en el estudio de Schlegel y colaboradores, referente a este punto de corte en cuanto al número de índices alterados.

En el trabajo de Martínez y Giraldo "Características topográficas corneales de los pacientes atendidos en la IPS Clínica de Optometría de la Universidad Santo Tomás de Colombia en el período comprendido entre el 19 de septiembre de 1997 a 19 de abril de 2006"11, sobre un total de 773 exámenes topográficos por primera vez encontraron que presen- taban ectasia en un $26,8 \%$ y $30,0 \%$ en ojo derecho e izquierdo, respectivamente, valor muy inferior al obtenido en el presente trabajo que fue del $47,9 \%$ en ojo derecho y del $52,1 \%$ en ojo izquierdo, sobre un total de 339 exámenes, es importante destacar que en el trabajo citado los datos obtenidos fueron con un topógrafo Tomey 2 que solo evalúa la cara anterior de la córnea, con el cual no se detectan los caso subclínicos de las ectasias corneales.

El índice Kprob (índice de probabilidad del queratocono) presenta una especificidad muy alta $(93,10 \%)$, lo cual permite decir que al referirse a la presencia de normalidad en el paciente su margen de error es muy bajo; mientras que la sensibilidad que presenta es muy baja (36,59\%), lo cual no es bueno en el momento para indicar al paciente la presencia de ectasia, por lo tanto no es confiable lo que arroja este índice.

\section{Conclusiones}

En el presente estudio (Sucre, Bolivia) se encontró una diferencia entre el género femenino $(57,6 \%)$ y masculino $(42,4 \%)$ del $15,2 \%$, dando como predominante el primero. En cuanto la edad su distribución muestra un rango comprendido entre los 7 y 64 años, dando mayor frecuencia a la edad de 30 años.

De las 339 topografias que se realizaron 279 tuvieron una excelente toma y 60 presentaron una calidad reducida debido a falta de anillos de plácido, sin embargo, el equipo los clasifica con un $97 \%$ de área analizada (normal).

La prevalencia de ectasias corneales en la Clínica Oftalmológica Solex de Sucre, fue del 57,2\%. En ojo derecho $47,9 \%$ y del $52,1 \%$ en ojo izquierdo sobre un total de 339 exámenes, es importante destacar que es una prevalencia alta.

Del grupo clasificado con ectasia, se encontró que el $62,4 \%$ de estas correspondían a ectasia incipiente, vale la pena anotar que en este grupo se encuentran los queratoconos frustros, los cuales no se clasificaron aparte, ya que solo se tenía para ella la edad, sin seguimiento clínico que permitiera clasificarlos como tal.

Se determina en el presente estudio que hubo más casos de ectasias corneales para género femenino con un $66 \%$ sobre el masculino con un 34\%. Pero es importante resaltar que en el estudio hay más personas del género femenino.

Se establece que no hay diferencia significativa en la edad entre el grupo normal y con queratocono.

Con respecto a los índices de elevación anterior como posterior se establecen valores alterados para 
ambos casos; donde también se determina que hubo diferencias significativas entre el grupo normal y de queratocono.

\section{BIBLIOGRAFÍA}

1. Wilson SE, Yu Guang HE, Weng J, McDowall AW, Vital M, Chwang EL. Epithelial Injury Induces KeratocyteAapoptosis: Hypothesized Role for the Interleukin-1 System in the Modulation of Corneal Tissue Organization and Wound Healing. Exp Eye Res 1996; 662$): 327-337$.

2. Krachmer HJ, Palay AD. Atlas de la Córnea, $2^{\mathrm{a}}$. ed. Madrid, España: Editorial Elsevier; 2008.

3. Galvis V, Tello A. Guías de oftalmología básica y clínica FOSCAL, $2^{\mathrm{a}}$. ed. Bucaramanga, Colombia. 2009, 147-155.

4. Enfermedades producidas por radiaciones ultravioletas [internet] 2011 abr [citado 2012 oct]; [aprox $1 \mathrm{p}$ ] disponible en http://ism-05-ism.blogspot.com/2011/04/ enfermedades-producidas-por-radiaciones.html.

5. Wilson SE, Yu Guang HE, Weng J, McDowall AW, Vital M, Chwang EL. Epithelial Injury Induces KeratocyteAapoptosis: Hypothesized Role for the Interleukin-1 System in the Modulation of Corneal Tissue Organization and Wound Healing. Exp Eye Res. 1996;\{62):327 37.

6. Ministerio de Salud Bolivia. Código de Ética y Deontología Médica [citado 2014 mar 4]; 21p. Disponible en http://www.minsalud.gob.bo/images/Documentacion/ normativa/CODIGODEETICAYDEONTOLOGIAMEDICA.pdf.
7. García R. Estudio retrospectivo sobre 290 casos de queratótomo. Gaceta Óptica. 2009 oct; 441:20-23.

8. Samara A, Benítez MC, Díaz Y, Machado E. Características topográficas del queratocono en nuestro medio. Rev Cubana Oftalmol [Internet]. 2003 dic;16(2):[aprox 7 p.] Disponible en: http://scielo.sld.cu/scielo.php? script = sci arttext\&pid=S0864-21762003000200008\&ln g=es

9. Fam H, Lim K. Corneal elevation indices in normal and keratoconic eyes. J Cataract Refract Surg. 2006;32:12811287.

10. Schlegel Z, Hoang-Xuan T, Gatinel D. Comparison of and correlation between anterior and posterior corneal elevation maps in normal eyes and keratoconus suspect eyes. J Cataract Refract Surg. 2008;34:789-795.

11. Martínez A, Giraldo M. Características topográficas corneales de los pacientes atendidos en la IPS Clínica de Optometría de la Universidad Santo Tomás en el periodo comprendido entre el 19 de septiembre de 1997 y el 19 de abril de 2006. [Trabajo de grado]. Bucaramanga, Colombia: Universidad Santo Tomás; 2008.

Correo electrónico de los autores:

Álvaro Ernesto Martínez Izquierdo: alvaro-emi@hotmail.com Freddy Guerra Paniagua:fred_war@yahoo.com Glynka María Guerra Torrico: kitty g22@hotmail.com Judith Alicia Cárdenas Remolina: jacr140119@hotmail.com Kerly Dayana Ibáñez Felizzola: No disponible.

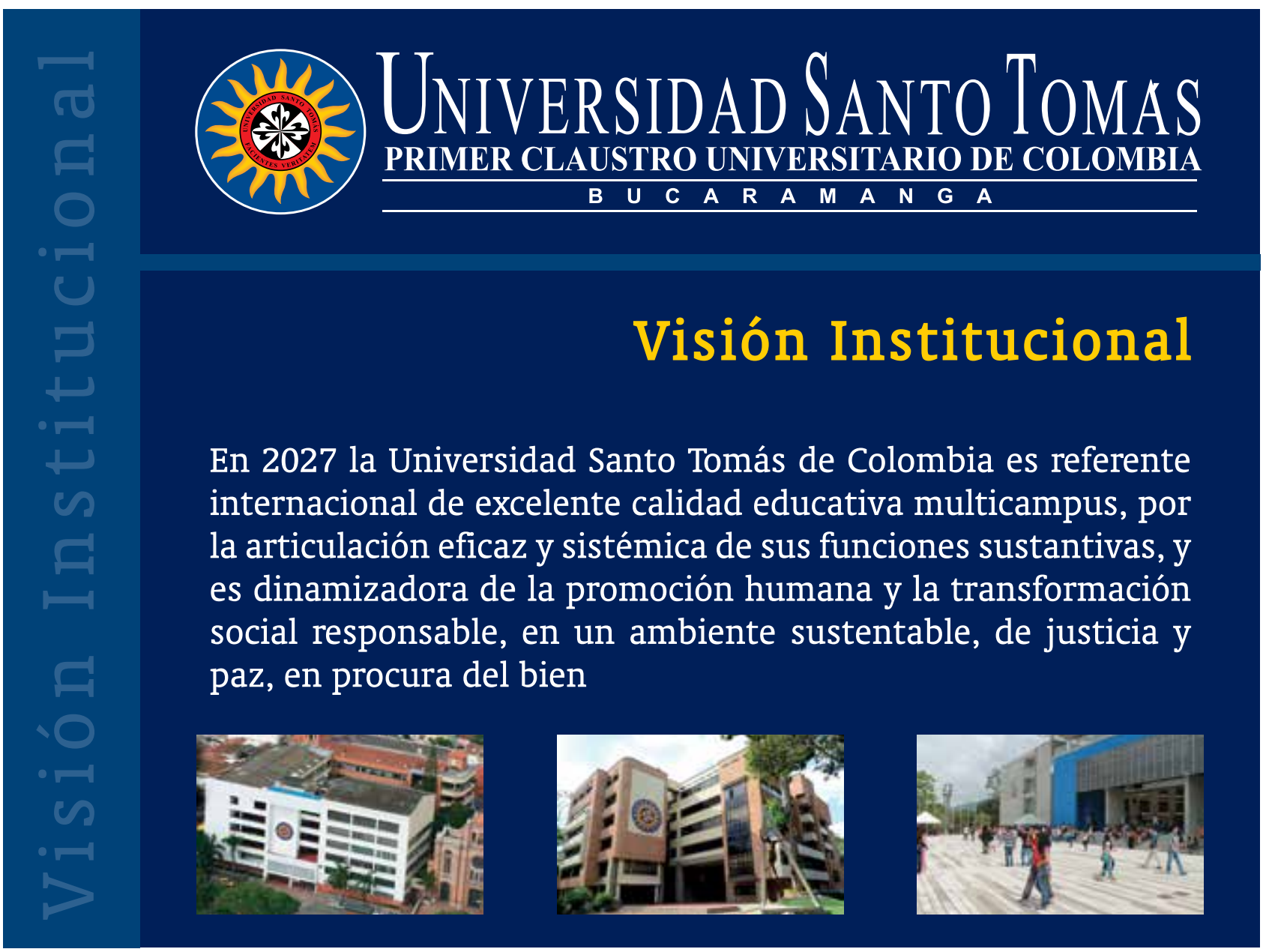

